\title{
Hemorrhagic atrial myxoma causing a cardioembolic stroke: a case report
}

\begin{abstract}
Identifying the etiology of an ischemic stroke is essential to the treatment and prevention of future events. Cardioembolic strokes occur in about $20 \%$ of stroke patients with atrial fibrillation being the most common cause $(50 \%)$ while myxomas account for only $0.5 \%$ of cases. This case demonstrates the successful use of TTE followed by TEE in identifying the etiology of this patient's stroke.
\end{abstract}

Keywords: Atrial myxoma, Cardioembolic stroke, Transthoracic echocardiogram, Transesophageal echocardiogram

\author{
Volume 2 Issue I - 2015 \\ Jessica Weiss DO,' Dave Beutler DO, Jason \\ Klein MD ${ }^{2}$ \\ 'Internal Medicine Residency Program, Banner Good Samaritan \\ Medical Center, USA \\ ${ }^{2}$ Cardiology Fellowship Program, Banner Good Samaritan \\ Medical Center, USA
}

\begin{abstract}
Correspondence: Jessica Weiss DO, Internal Medicine Residency Program, Banner Good Samaritan Medical Center, I I I E. McDowell Rd, Phoenix, AZ 856, USA, Tel (8I8) 2039064, Fax (602) 839-2084, Email wjessica I 16@gmail.com
\end{abstract}

Received: September 03, 2014 | Published: January 16, 2015
Abbreviations: TTE, Transthoracic Echocardiogram; TEE, Transesophageal Echocardiogram; MRI, Magnetic Resonance Imaging

\section{Introduction}

Cardioembolic strokes occur in about $20 \%$ of stroke patients, with atrial fibrillation being the most common cause (50\%), while myxomas account for only $0.5 \%$ of case. ${ }^{1,2}$ Identifying the etiology of an ischemic stroke is essential to the treatment and prevention of future events. Echocardiography an essential diagnostic tool used to identify a potential cardioembolic source of an ischemic stroke. The following case presentation demonstrates the successful use of transthoracic echocardiogram (TTE) followed by transesophageal echocardiogram (TEE) in identifying a cardiac myxoma as the etiology of an acute ischemic stroke.

\section{Case report}

A 71-year-old African American female with history of hypertension, dyslipidemia, diabetes mellitus and end-stage renal disease presented with acute onset of visual changes, severe headache, dizziness and nausea. Physical exam was significant for an elevated blood of $165 / 73$ with a heart rate of 79 , a right upper quadrant visual field deficit and an II/VI systolic murmur but was otherwise unremarkable. Carotid Doppler ultrasound showed mild mixed plaque in the bilateral carotid bulbs and proximal internal and external carotid arteries without hemodynamically significant stenosis. Head computed tomography negative for acute changes, however did reveal a $2 \mathrm{~cm}$ sellar/suprasellar mass. Brain magnetic resonance imaging showing acute ischemia in the left middle cerebral artery territory, suggestive of an embolic source (Figure 1). Additionally the MRI was able to better characterize the $2.4 \mathrm{~cm}$ sellar mass as most likely resembling a pituitary macroadenoma (likely explaining the quadrantanopia). The brain MRI prompted a TTE, which disclosed a mass associated with the posterior mitral annulus (Figure 2). Subsequent TEE showed a highly mobile, $1.5 \times 1.0 \mathrm{~cm}$ pedunculated smooth-appearing mass located the near posterior mitral valve leaflet attached with a thin stalk. The mass prolapsed into the left ventricle during diastole. The TEE study showed no left atrial appendage thrombus and was negative for an interatrial shunt. The patient underwent surgical removal of the left atrial mass. Pathologic examination revealed a $1.5 \times 1.0$ x $0.9 \mathrm{~cm}$ benign hemorrhagic atrial myxoma (Figure 3).

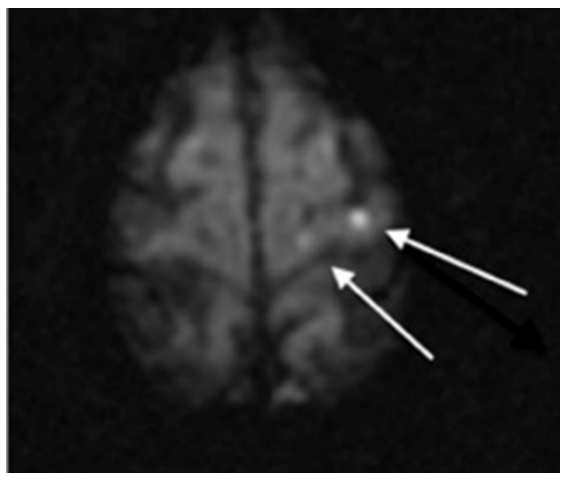

Figure I Brain MRI showing acute ischemia in the left middle cerebral artery territory, special the left convexity within the precentral gyrus, suggestive of an embolic source.

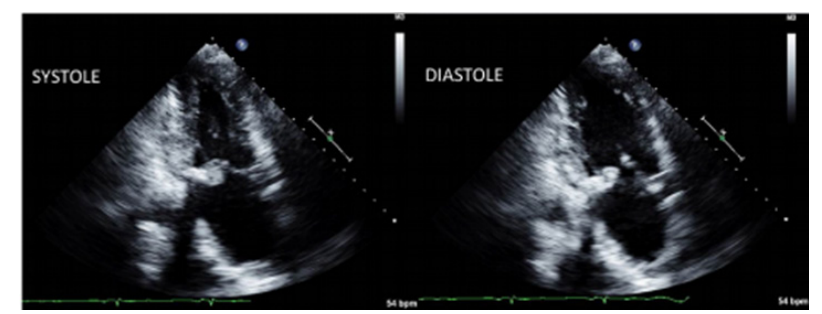

Figure 2 Transthoracic echocardiogram showing a mass associated with the posterior mitral annulus.

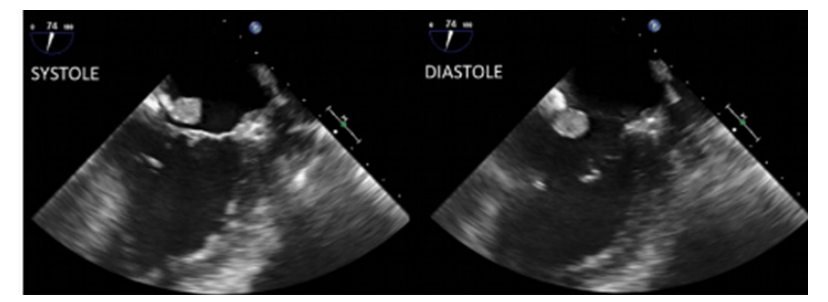

Figure 3 Transesophageal echocardiogram showing a highly mobile, 1.5 × 1.0 $\mathrm{cm}$ pedunculated smooth-appearing mass located the near posterior mitral valve leaflet attached with a thin stalk with the mass prolapsing into the left ventricle during diastole. 


\section{Discussion}

Cardiac myxomas, although exceedingly rare (incidence $0.001-$ $0.3 \%$ ), are associated with a high rate of embolization (30-40\%). ${ }^{1-5}$ Characteristically benign ( $75 \%$ benign, $25 \%$ malignant), cardiac myxomas are the most common cardiac tumor in adults, often occurring in the third to sixth decade of life with a female to male ratio of $2-3: 1{ }^{2,4,5}$ The majority $(90 \%)$ of atrial myxomas are sporadic with a minority $(10 \%)$ being familial secondary to an autosomal dominant disease known as Carney complex. ${ }^{2,4}$ Cardiac myxomas most commonly originate in the left atrium (75\%) and the most frequent implantation site is the atrial septum near the fossa ovalis. ${ }^{2,5,6}$ Tumor size varies between $1-15 \mathrm{~cm}$ with a mean tumor diameter of $2.7 \pm 1.3 \mathrm{~cm}$. Histologically, myxomas originate from mesenchymal cells of the septal endocardium, are gelatinous with a smooth or lobulated surface, usually white, yellow or brown in colorand can be villous, papillary, sessile or pedunculated..$^{2,4,7}$ The pedunculated type accounts for approximately half of the cases and are the most likely to cause emboli due to their mobility. ${ }^{7}$ Although embolization can occur is multiple different organ systems, cerebral infarctions are the most common and the MCA is the predominant area affected. ${ }^{4,8}$ Given the significant embolic consequences of atrial myxomas, such as embolic, hemorrhagic or recurrent cerebral infarctions, progressive multi-infarct dementia and event massive embolic stroke leading to sudden death, ${ }^{2}$ it is crucial to quickly diagnose and treat in order to circumvent future catastrophic events.

Echocardiography is the gold standard for diagnosis. The 2009 AHA/ASA guidelines state that echocardiography indicated when no cause for TIA or ischemic stroke has been identified by initial work up. ${ }^{9}$ Usually TTE is utilized first as it is less invasive and better tolerated. Transesophageal echocardiogram is useful when TTE findings are negative or ambiguous and is the best test to diagnose the presence of a left atrial appendage thrombus, left ventricular thrombi in patients with heart failure or previous myocardial infarction, aortic arch atherosclerosis, patent foramen ovale, atrial septal aneurysm, valvular disease or cardiac myxomas. Transthoracic echocardiogram, although less invasive, is less sensitive in comparison to TEE for diagnosis the aforementioned cardiac sources of emboli.

Definitive treatment is prompt surgical excision of the myxoma, which has shown to have excellent early and long-term results. Operative death rate in one care series reported a death rate of $<5 \%{ }^{5}$ Given a recurrence rate of about $1-3 \%$, patients should be monitored annually with echocardiography for several years after resection. ${ }^{2,10}$
This case demonstrates that although cardiac myxomas are a rare cause of ischemic stroke, the use of echocardiography, both TTE and TEE, are essential to the diagnostic work up of ischemic stroke.

\section{Acknowledgments}

None.

\section{Conflicts of interest}

None.

\section{References}

1. Reynen K. Cardiac myxomas. N Engl J Med. 1995;333(24):1610-1617.

2. Akhtar J, Wasay M, Rauf J. Atrial myxoma: a rare cause of cardioembolic stroke. BMJ Case Reports 2012. 2012;doi:10.1136/bcr.2012.006176.

3. Wein TH, Bornstein NM. Stroke Prevention: Cardiac and CarotidRelated Stroke. Neurol . 2000;18(2):321-341.

4. Yoo M, Graybeal DF. An echocardiographic-confirmed case of atrial myxoma causing cerebral embolic ischemic stroke: a case report. Cases J. 2008;1(1):96.

5. Garatti A, Nano G, Canziani A, et al. Surgical excision of cardiac myxomas: twenty years experience at a single institution. Ann Thorac Surg. 2012;93(3):825-831.

6. Ozaydin M, Dogan A, Altinbas A. Left atrial myxoma presenting with acute myocardial infarction -a case report. Angiology. 2005;56(6):767769

7. Rescigno G, Matteucci SL, D'Alfonso A, et al. A cryptogenic pulmonary embolism: left atrial myxoma occluding an atrial septal defect. J Thorac Cardiovasc Surg. 2006;132(5):1223-1224.

8. Hofmann E, Becker T, Romberg-Hahnloser R, et al. Cranial MRI and CT inpatients with left atrial myxoma. Neuroradiology. 1992;34(1):5761.

9. Easton JD, Saver JL, Albers GW, et al. Definition and evaluation of transient ischemic attack: a scientific statement for healthcare professionals from the American Heart Association/American Stroke Association Stroke Council; Council on Cardiovascular Surgery and Anesthesia; Council on Cardiovascular Radiology and Intervention; Council on Cardiovascular Nursing; and the Interdisciplinary Council on Peripheral Vascular Disease. The American Academy of Neurology affirms the value of this statement as an educational tool for neurologists. Stroke. 2009;40(6):2276-2293

10. O’Rourke F, Dean N, Mouradian MS, et al. Atrial myxoma as a cause of stroke: case report and discussion. CMAJ. 2003;169(10):1049-1051. 